Article

\title{
A New Equation to Estimate Energy Expenditure Using Heart Rate in Children
}

\author{
Mihyun Lee ${ }^{1} \mathbb{D}$, Jeong-Hui Park ${ }^{2} \mathbb{D}$, Myong-Won Seo ${ }^{3} \mathbb{D}$, Seoung-Ki Kang ${ }^{4}$ and Jung-Min Lee ${ }^{2, *(\mathbb{D})}$ \\ 1 Department of Physical Education, Sungkyul University, 53, Seonggyeoldaehak-ro, Manan-gu, \\ Anyang-si 14097, Korea; ksme_1998@naver.com \\ 2 Department of Physical Education, Kyung Hee University (Global Campus), 1732 Deokyoungdaero, \\ Giheung-gu, Yongin-si 17014, Korea; jeonghee@khu.ac.kr \\ 3 Department of Taekwondo, Kyung Hee University (Global Campus), 1732 Deokyoungdaero, Giheung-gu, \\ Yongin-si 17014, Korea; myongwonseo@khu.ac.kr \\ 4 Graduate School of Education, Yongin University, 134 Yongindaehak-ro, Cheoin-gu, Yongin-si 449-714, Korea; \\ ksk0527@hanmail.net \\ * Correspondence: jungminlee@khu.ac.kr; Tel.: +82-31-201-2736
}

Citation: Lee, M.; Park, J.-H.; Seo, M.-W.; Kang, S.-K.; Lee, J.-M. A New Equation to Estimate Energy Expenditure Using Heart Rate in Children. Sustainability 2021, 13, 5092. https://doi.org/10.3390/su13095092

Academic Editors: Guillermo Felipe López Sánchez and Lee Smith

Received: 16 April 2021

Accepted: 29 April 2021

Published: 1 May 2021

Publisher's Note: MDPI stays neutral with regard to jurisdictional claims in published maps and institutional affiliations.

Copyright: (c) 2021 by the authors. Licensee MDPI, Basel, Switzerland. This article is an open access article distributed under the terms and conditions of the Creative Commons Attribution (CC BY) license (https:/ / creativecommons.org/licenses/by/ $4.0 /)$.

\begin{abstract}
The first aim of this study was to develop equations to predict physical activity energy expenditure (PAEE) for children utilizing heart rate monitors (HRM) and vector magnitudes (VM) from accelerometers. The second aim was to cross-validate the developed PAEE prediction equations and compare the equations to the pre-existing accelerometer-based PAEE equation (i.e., Trost). Seventy-five students in elementary school (from 10 to 13 years old) were classified into an equation calibration group ( $\mathrm{N}=50,33$ boys and 17 girls) and a cross-validation group $(\mathrm{N}=25,20$ boys and 5 girls). Participants simultaneously wore a portable indirect calorimeter (Cosmed's $K 4 b^{2}$ ), a heart rate monitor on the chest, and an accelerometer on the right side of the waist. Then, the participants performed a series of various intensity activities. The energy expenditure (EE) measured by $K 4 b^{2}$ was set as the dependent variable. Multiple regression analysis was performed to derive the heart rate and accelerometer-based equations. The heart-rate-based EE equation had an explanatory power of adj. $R^{2}=0.814$ and the accelerometer-based EE equation had an explanatory power of adj. $R^{2}=0.802$. The VM-based EE indicated high mean absolute percent errors (MAPE) at light, moderate, and vigorous intensity. The heart-rate-based EE was included in the range of equivalence limit in all activities, but the VM and pre-existing equation showed some overestimation beyond the equivalence range. The agreement errors between the criterion EE and the estimated EE were lower in the heart-rate-based equation than the accelerometer-based equations (i.e., VM and Trost). The approach with the heartrate-based EE equation demonstrated higher accuracy than the accelerometer-based EE equations. The results of the current study indicate that the heart-rate-based PAEE equation can be a potential method for estimating children's PAEE.
\end{abstract}

Keywords: children; energy expenditure; heart rate; accelerometer

\section{Introduction}

Children's participation in physical activities (PA) plays a critical role in promoting their lifelong health and well-being and in preventing risk factors for health conditions such as heart disease, obesity, and type 2 diabetes [1-4]. In the 2018 Physical Activity Guidelines for Americans, children and adolescents aged 6 to 17 were encouraged to perform moderate to vigorous aerobic intensity PA for at least 60 min daily [5]. Many previous studies have demonstrated that adolescents who participate more in PA that meet guidelines and spend less time sitting are much healthier physically and mentally than those who do not [6-8]. Furthermore, some studies have found that adolescents who participate more in PA have a lower risk of disease exposure and better sleep quality than others $[9,10]$. To identify the association between actual health outcomes and the 
compliance to meet the daily recommended PA for children, objective and accurate PA assessment for children and adolescents is essential in a free-living environment.

Among the PA assessment tools, the gold standard methods such as doubly labeled water and indirect calorimetry are used to measure energy expenditure (EE). However, they tend to be costly, require a specific place, and are difficult to use, limiting their feasibility in extensive population studies despite their capability to measure the EE accurately. For these reasons, field test instruments such as accelerometers and heart rate monitors (HRM) are convenient and are predominantly used in extensive cohort studies at a relatively low cost [11-13].

The accelerometer estimates physical activity energy expenditure (PAEE) using an equation developed based on the values (i.e., counts). Many types of accelerometer devices, especially ActiGraph (ActiGraph, Pensacola, FL, USA), have been utilized to estimate the PAEE in free-living conditions [14]. Studies using ActiGraph have been conducted extensively, and ActiGraph has also been used in large-scale national studies of children and adolescents [15]. Furthermore, studies examining the validity and reliability of accelerometers have been published by many researchers [16-19], but most of these previous studies investigated equations for classifying PA intensity by applying cut-off points. There have been limited studies on estimating the PAEE equation and few studies have examined the prediction accuracy of the PAEE estimates using accelerometers in children [20,21]. Furthermore, HRMs have been used longer as a tool to measure PA levels because of their ease of use and accurate estimation of PAEE made possible based on the fact that the heart rate during PA has a strong linear relationship with oxygen uptake [22]. However, EE prediction using HRMs has been reduced for a while due to the poor comfort of fit.

Advanced technology integrating HR and accelerometers has been developed to estimate PAEE (i.e., Actiheart) in adults and children; in particular, one study indicated that PAEE estimates in children using the Actiheart were not valid compared to the indirect calorimetry [23]. In addition, one of the PA monitoring companies has developed a device to estimate EE to combine the information from triaxle accelerometers, galvanic skin sensors, and heat flux sensors (i.e., Sensewear Armband). The device has proven its validity of estimating EE compared to the doubly labeled water in children [24], but the device is no longer available. Therefore, it is necessary to develop a valid PAEE prediction equation for researchers and health practitioners to apply the simple and feasible PAEE estimates in children. Therefore, this study's first aim was to develop two EE equations, i.e., (1) heart-rate-based PAEE and (2) accelerometer (i.e., vector magnitude)-based PAEE in children. The second aim of the study was to cross-validate the developed PAEE estimate equations compared to the criterion, and an additional exploratory comparison was made with commonly used accelerometer-based PAEE equations (i.e., Trost equation).

\section{Methods}

\subsection{Study Participants}

This study was approved by the Institutional Review Board (IRB). This study's participants were selected through convenience sampling, with 75 children aged 10 to 13 years old who lived in South Korea. Table 1 presents the demographic characteristics of participants. A total of 33 boys (age: $10.88 \pm 1.11$ years, weight: $38.36 \pm 5.99 \mathrm{~kg}$, height: $140.48 \pm 6.89 \mathrm{~cm}$ ) and 17 girls (age: $11.47 \pm 0.87$ years, weight: $41.71 \pm 9.98 \mathrm{~kg}$, height: $143.17 \pm 6.21 \mathrm{~cm}$ ) were in the equation calibration group. For the cross-validation group, 21 boys (age: $11.14 \pm 1.01$ years, weight: $37.71 \pm 5.87 \mathrm{~kg}$, height: $143.12 \pm 6.48 \mathrm{~cm}$ ) and 4 girls (age: $11.50 \pm 0.58$ years, weight: $47.50 \pm 10.38 \mathrm{~kg}$, height: $145.15 \pm 1.70 \mathrm{~cm}$ ) were used to validate the prediction equation models. Prior to the study, the study purpose was fully described in detail to parents and participants. Eligible participants voluntarily gave their consent to participate in the study; informed parental consent and child assent were obtained. 
Table 1. Characteristics of the participants (mean \pm SD).

\begin{tabular}{ccccccc}
\hline & \multicolumn{2}{c}{$\begin{array}{c}\text { Total } \\
(\mathbf{n}=\mathbf{7 5})\end{array}$} & \multicolumn{2}{c}{$\begin{array}{c}\text { Calibration Group } \\
(\mathbf{n}=\mathbf{5 0})\end{array}$} & \multicolumn{2}{c}{$\begin{array}{c}\text { Cross Validation Group } \\
(\mathbf{n}=\mathbf{2 5})\end{array}$} \\
\cline { 2 - 7 } Variable & $\begin{array}{c}\text { Boys } \\
(\mathbf{n}=\mathbf{5 4 )}\end{array}$ & $\begin{array}{c}\text { Girls } \\
(\mathbf{n}=\mathbf{2 1})\end{array}$ & $\begin{array}{c}\text { Boys } \\
(\mathbf{n}=\mathbf{3 3})\end{array}$ & $\begin{array}{c}\text { Girls } \\
(\mathbf{n}=\mathbf{1 7})\end{array}$ & $\begin{array}{c}\text { Boys } \\
(\mathbf{n}=\mathbf{2 1})\end{array}$ & $\begin{array}{c}\text { Girls } \\
(\mathbf{n}=\mathbf{4})\end{array}$ \\
\hline Age (year) & $11.01 \pm 1.06$ & $11.49 \pm 0.73$ & $10.88 \pm 1.11$ & $11.47 \pm 0.87$ & $11.14 \pm 1.01$ & $11.50 \pm 0.58$ \\
Height $(\mathrm{cm})$ & $141.80 \pm 6.69$ & $144.16 \pm 3.96$ & $140.48 \pm 6.89$ & $143.17 \pm 6.21$ & $143.12 \pm 6.48$ & $145.15 \pm 1.70$ \\
Weight $(\mathrm{kg})$ & $38.04 \pm 5.93$ & $44.60 \pm 10.18$ & $38.36 \pm 5.99$ & $41.71 \pm 9.98$ & $37.71 \pm 5.87$ & $47.50 \pm 10.38$ \\
BMI $\left(\mathrm{kg} \cdot \mathrm{m}^{-2}\right)$ & $18.90 \pm 2.46$ & $21.45 \pm 4.84$ & $19.45 \pm 2.85$ & $20.29 \pm 4.38$ & $18.34 \pm 2.07$ & $22.61 \pm 5.30$ \\
\hline
\end{tabular}

SD: standard deviation, BMI: body mass index; There are no significant differences in age, height, weight, and BMI between the calibration group and the cross-validation group.

\subsection{Instrument}

\subsubsection{Cosmed's $K 4 b^{2}$ (COSMED, Rome, Italy)}

The criterion EE was measured by Cosmed's $K 4 b^{2}$ (COSMED, Rome, Italy). $K 4 b^{2}$ is a portable indirect calorimetry method that allows measurement of real-time energy expenditure and has been widely used as a criterion method in measuring PA levels of children and adolescents and adults [11,25-27]. The lightweight (925g) K4b ${ }^{2}$ worn on the back can monitor $\mathrm{VO}_{2}, \mathrm{VCO}_{2}$, and energy expenditure without having to be inside a laboratory. Calibration was performed according to the manufacturer's instructions before starting the experiment.

\subsubsection{POLAR S810 (Polar Electro, Kempele, Finland)}

For the HRM, a POLAR S810 (Polar Electro, Kempele, Finland) was used. The POLAR S810 has demonstrated high validity in measuring children's heart rates [28]. The POLAR heart rate strap was worn tightly against the skin just below the pectoral muscles.

\subsubsection{The ActiGraph GT3X+ (ActiGraph Corp, Pensacola, FL, USA)}

For the accelerometer, the GT3X+ (ActiGraph, Pensacola, FL, USA) ActiGraph was used. The GT3X+ accelerometer is a compact $(3.8 \mathrm{~cm} \times 3.7 \mathrm{~cm} \times 1.8 \mathrm{~cm})$, lightweight $(20 \mathrm{~g})$ triaxial accelerometer. The ActiGraph accelerometer is used for research purposes and in many national studies of children and adolescents [15,29-31]. When measuring, the accelerometer is fixed using an elastic belt over the right hip. The accelerometer's data period was set to five seconds based on a previous study by Trost et al. [20]. It was initialized using ActiLife- 6 software before the start of the experiment, and after all activities were completed, the activity count data were downloaded and converted to Microsoft Excel for analysis. The counts per minute (CPM) of the vertical axis (VT) and vector magnitudes $\left(V M=\sqrt{x^{2}+y^{2}+z^{2}}\right)$ of the GT3X+ were analyzed.

\subsection{Study Protocol}

Height was measured and recorded in units of $0.1 \mathrm{~cm}$ with an extensometer (Jenix, Seoul, Korea) and body weight was recorded in units of $0.1 \mathrm{~kg}$ with an electronic scale (CAS, Seoul, Korea). The body mass index (BMI) was calculated by dividing the formula weight $(\mathrm{kg})$ by the square of the height $\left(\mathrm{m}^{2}\right)$. Measurements were conducted in an indoor gym equipped with a treadmill. Eating and exercise were prohibited at least three hours before the start of the experiment to prevent further variability in energy expenditure that could affect the study results. The experimental protocol was selected based on the activities used in previous studies on children's PA [11,12,17,18]. Participants contributed to the two data collection points on a two-week cycle for the collection of $\mathrm{VO}_{2}$, heart rate, and accelerometer data. At the same time, they performed a series of activities according to the protocol (i.e., light-intensity: slow walking at $2 \mathrm{~km} / \mathrm{h}$ and throwing darts; moderateintensity: normal walking at $4 \mathrm{~km} / \mathrm{h}$, fast walking at $6 \mathrm{~km} / \mathrm{h}$, and ball catching; and vigorous-intensity: running at $8 \mathrm{~km} / \mathrm{h}$ and hopscotch). For the first visit, height and weight were measured, and then the participants rested quietly for five minutes wearing $K 4 b^{2}$, 
the HRM, and accelerometers for restful breathing. Participants randomly performed two treadmill activities: (1) normal walking at $4 \mathrm{~km} / \mathrm{h}$ and (2) running at $8 \mathrm{~km} / \mathrm{h}$ on the treadmill. Participants were asked to perform slow walking at $2 \mathrm{~km} / \mathrm{h}$ and fast walking at $6 \mathrm{~km} / \mathrm{h}$ on the treadmill for the second visit. Regarding the cross-validation group, participants were asked to perform additional activities of throwing darts, ball catching, and hopscotch on the second day, which are children's usual free-living activities. All activities were conducted for five minutes, and there was a five-minute break between each activity.

\subsection{Data Analysis}

All results obtained from the study used SPSS 25.0 version (IBM SPSS, Chicago, IL, USA) for Windows and MedCalc ver. 14.0 (MedCalc, Belgium, UK) for analysis. Descriptive statistics were applied to calculate the mean and standard deviation of physical characteristics of the collected data. Personal information and physiological variables frequently used as independent variables in previous studies were selected as common variables, such as age, gender, height, and weight [32]. Since gender is a categorical variable, it was used as a dummy variable for input rather than directly input as an independent variable for stepwise multiple regression analysis. For the optimal regression model's variable selection method, the coefficient of determination was corrected with the statistics and selection method, and a Monte Carlo cross-validation procedure was used. To evaluate the accuracy of predicted EE using the three methods, the error difference from the predicted EE was calculated using Mean Absolute Percent Errors (MAPE) in comparison with indirect calorimetry (i.e., $K 4 b^{2}$ ). For the estimated errors between the criterion EE and predicted $\mathrm{EE}$, the general agreement across the range of estimated $\mathrm{EE}$ values with corresponding $\pm 95 \%$ limit of agreement (LoA) with fitted lines (from regression analyses between mean and difference) was estimated using the Bland and Altman graphical procedure with their corresponding parameters (i.e., intercept, slope). Equivalence testing was performed to examine the equivalence between the criterion EE and the predicted EE. Regarding the equivalence test, when the $90 \%$ confidence intervals (CI) for the mean of the predicted EE falls within the proposed equivalence limit $( \pm 10 \%)$ range of the criterion $\mathrm{EE}$, then the value was equivalent to the criterion measurement values. The statistical significance was set at $p<0.05$.

\section{Results}

Table 2 presents the mean and standard deviation of the criterion $\mathrm{VO} 2, \mathrm{EE}$, heart rate, and accelerometer count (i.e., vector magnitude) for each activity intensity in the children's EE equation calibration group $(\mathrm{N}=50)$. The criterion EE measurement device (i.e., $\left.K 4 b^{2}\right)$ gradually increased as activity intensity increased. Specifically, the values measured $\mathrm{VO}_{2}$ at $2 \mathrm{~km} / \mathrm{h}$ were higher in boys than girls; however, the $\mathrm{VO}_{2}$ values were higher in girls than boys at $6 \mathrm{~km} / \mathrm{h}$ and $8 \mathrm{~km} / \mathrm{h}$. In rest, the boys indicated higher values of $\mathrm{VO}_{2}$ and $\mathrm{EE}$ than girls, whereas values of HR (boy: $89.80 \pm 11.94$ beats/min, girl: $91.17 \pm 12.00$ beats/min) and VM (boy: $114.48 \pm 207.47$, girl: $276.64 \pm 217.46$ ) were opposite compared to $\mathrm{VO} 2$ and EE. Furthermore, $\mathrm{VO}_{2}$ at $4 \mathrm{~km} / \mathrm{h}$ was measured higher in boys than in girls at $20.85 \pm 4.36$ and $19.75 \pm 4.68 \mathrm{~mL} / \mathrm{kg} / \mathrm{min}$, respectively; however, EE (boy: $3.66 \pm 1.08$, girl: $3.68 \pm 1.14 \mathrm{kcal} / \mathrm{min}$ ), HR (boy: $123.66 \pm 11.97$, girl: $128.18 \pm 10.25$ beat $/ \mathrm{min}$ ), and VM (boy: $2604.21 \pm 735.84$, girl: $2718.88 \pm 750.96$ counts $/ \mathrm{min}$ ) were measured higher in girls than boys. 
Table 2. Descriptive statistics of the activity intensity (mean $\pm \mathrm{SD}$ ). SD: standard deviation, $\mathrm{VO}_{2}$ : oxygen consumption, EE: energy expenditure, HR: heart rate, VM: vector magnitude.

\begin{tabular}{|c|c|c|c|c|c|}
\hline \multicolumn{2}{|c|}{ Variables } & \multirow{2}{*}{$\begin{array}{c}\mathrm{VO}_{2} \\
\left(\mathbf{m L} \cdot \mathbf{k g} \cdot \mathbf{m i n}^{-1}\right)\end{array}$} & \multirow{2}{*}{$\begin{array}{c}\begin{array}{c}\mathrm{EE} \\
\left(\mathbf{k c a l}^{\prime} \mathbf{m i n}^{-\mathbf{1}}\right)\end{array} \\
1.12 \pm 0.38\end{array}$} & \multirow{2}{*}{ 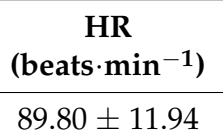 } & \multirow{2}{*}{$\begin{array}{c}\text { VM } \\
{\text { (counts } \cdot \text { min }^{-1} \text { ) }}^{\text {(con }} \\
114.48 \pm 207.47\end{array}$} \\
\hline Resting & Boy & & & & \\
\hline Kesting & Girl & $5.51 \pm 1.49$ & $1.06 \pm 0.27$ & $91.17 \pm 12.00$ & $276.64 \pm 217.46$ \\
\hline \multirow{2}{*}{$2 \mathrm{~km} / \mathrm{h}$} & Boy & $11.67 \pm 2.57$ & $2.00 \pm 0.62$ & $108.28 \pm 17.79$ & $1529.96 \pm 459.20$ \\
\hline & Girl & $10.84 \pm 2.93$ & $1.92 \pm 0.63$ & $105.23 \pm 12.47$ & $1415.19 \pm 660.47$ \\
\hline \multirow{2}{*}{$4 \mathrm{~km} / \mathrm{h}$} & Boy & $20.85 \pm 4.36$ & $3.66 \pm 1.08$ & $123.66 \pm 11.97$ & $2604.21 \pm 735.84$ \\
\hline & Girl & $19.75 \pm 4.68$ & $3.68 \pm 1.14$ & $128.18 \pm 10.25$ & $2718.88 \pm 750.96$ \\
\hline \multirow{2}{*}{$6 \mathrm{~km} / \mathrm{h}$} & Boy & $31.27 \pm 1.08$ & $5.52 \pm 1.54$ & $151.21 \pm 15.29$ & $4017.14 \pm 851.98$ \\
\hline & Girl & $32.21 \pm 5.66$ & $6.08 \pm 1.68$ & $159.21 \pm 13.69$ & $4490.49 \pm 1116.59$ \\
\hline \multirow{2}{*}{$8 \mathrm{~km} / \mathrm{h}$} & Boy & $42.24 \pm 8.39$ & $7.46 \pm 1.80$ & $173.27 \pm 16.51$ & $6710.95 \pm 1504.27$ \\
\hline & Girl & $42.46 \pm 7.14$ & $7.99 \pm 2.04$ & $178.16 \pm 11.42$ & $6888.26 \pm 1387.56$ \\
\hline
\end{tabular}

Table 3 represents the regression coefficients for both the HR-based equation and the VM-based equation in this study.

$$
\begin{aligned}
& \mathrm{EE}_{\mathrm{HR}}=-13.761-(0.461 \times \text { gender })+(0.242 \times \text { age })+(0.023 \times \text { height })+(0.075 \times \text { weight })+(0.069 \times \mathrm{HR}) . \\
& \mathrm{EE}_{\mathrm{VM}}=-1.033-(0.200 \times \text { gender })-(0.186 \times \text { age })-(0.002 \times \text { height })+(0.117 \times \text { weight })+(0.001 \times \mathrm{VM})
\end{aligned}
$$

where, gender is 0 for boy and 1 for girl and VM is calculated by counts per minute. The heart-rate-based equation is $\mathrm{F}=213.286(p<0.001)$ and the explanatory power is $81.8 \%\left(\right.$ adj $R^{2}=0.814, \mathrm{SEE}=1.171$, Durbin-Watson $\left.=1.886\right)$; the VM-based equation is $\mathrm{F}=196.805(p<0.001)$ and the explanatory power is $80.6 \%\left(\operatorname{adj} R^{2}=0.802, \mathrm{SEE}=1.210\right.$, Durbin-Watson $=1.902)$. The Trost et al. [20]-based equation $(\mathrm{EEm}=-2.23+(0.0008 \times \mathrm{VT}$, counts per minute $)+(0.08 \times$ weight $))$ was applied to compare the EE estimates between three EE predictive equations.

\begin{tabular}{|c|c|c|c|c|}
\hline \multirow{2}{*}{ Parameter } & \multicolumn{2}{|c|}{ Heart Rate Equation } & \multicolumn{2}{|c|}{ Vector Magnitude Equation } \\
\hline & Coefficients (95\% CI) & Standard Error & Coefficients (95\% CI) & Standard Error \\
\hline Intercept & $-13.761(-17.120$ to -10.402$)$ & 1.705 & $-1.033(-4.379$ to 2.313$)$ & 1.698 \\
\hline Gender & $-0.461(-0.787$ to -0.135$)$ & 0.165 & $-0.200(-0.536$ to 0.136$)$ & 0.171 \\
\hline Age (year) & $0.242(0.061$ to 0.424$)$ & 0.092 & $-0.186(-0.372$ to 0.000$)$ & 0.094 \\
\hline Height (cm) & $0.023(-0.006$ to 0.052$)$ & 0.015 & $-0.002(-0.032$ to 0.028$)$ & 0.015 \\
\hline Weight (kg) & $0.075(0.052$ to 0.099$)$ & 0.012 & $0.117(0.093$ to 0.141$)$ & 0.012 \\
\hline Heart Rate (beat/min) & $0.069(0.065$ to 0.073$)$ & 0.002 & & \\
\hline Vector Magnitude & & & $0.001(0.001-0.001)$ & 0.000 \\
\hline
\end{tabular}

Table 3. Regression coefficients for estimating PAEE with HR and VM.

SD: standard deviation, CI: confidence interval, SE: standard error, PAEE: physical activity energy expenditure, HR: heart rate, VM: vector magnitude.

Figure 1 illustrates the Mean Absolute Percent Errors (MAPE) calculated to examine the difference between the criterion $\mathrm{EE}$ and the predicted EE for the cross-validation group $(\mathrm{N}=25)$. The heart-rate-based EE showed MAPE at a $20 \%$ level in moderateintensity activities and vigorous-intensity activities and somewhat higher MAPE in lightintensity activities (38.56\%). The VM-based EE showed MAPE values in the order of moderate-intensity activities (30.14\%), vigorous-intensity activities $(41.73 \%)$, and lightintensity activities (47.02\%). The Trost-based equations showed the MAPE values in the order of vigorous-intensity activities $(21.3 \%)$, moderate-intensity activities (38.44\%), and light-intensity activities (47.02\%). 


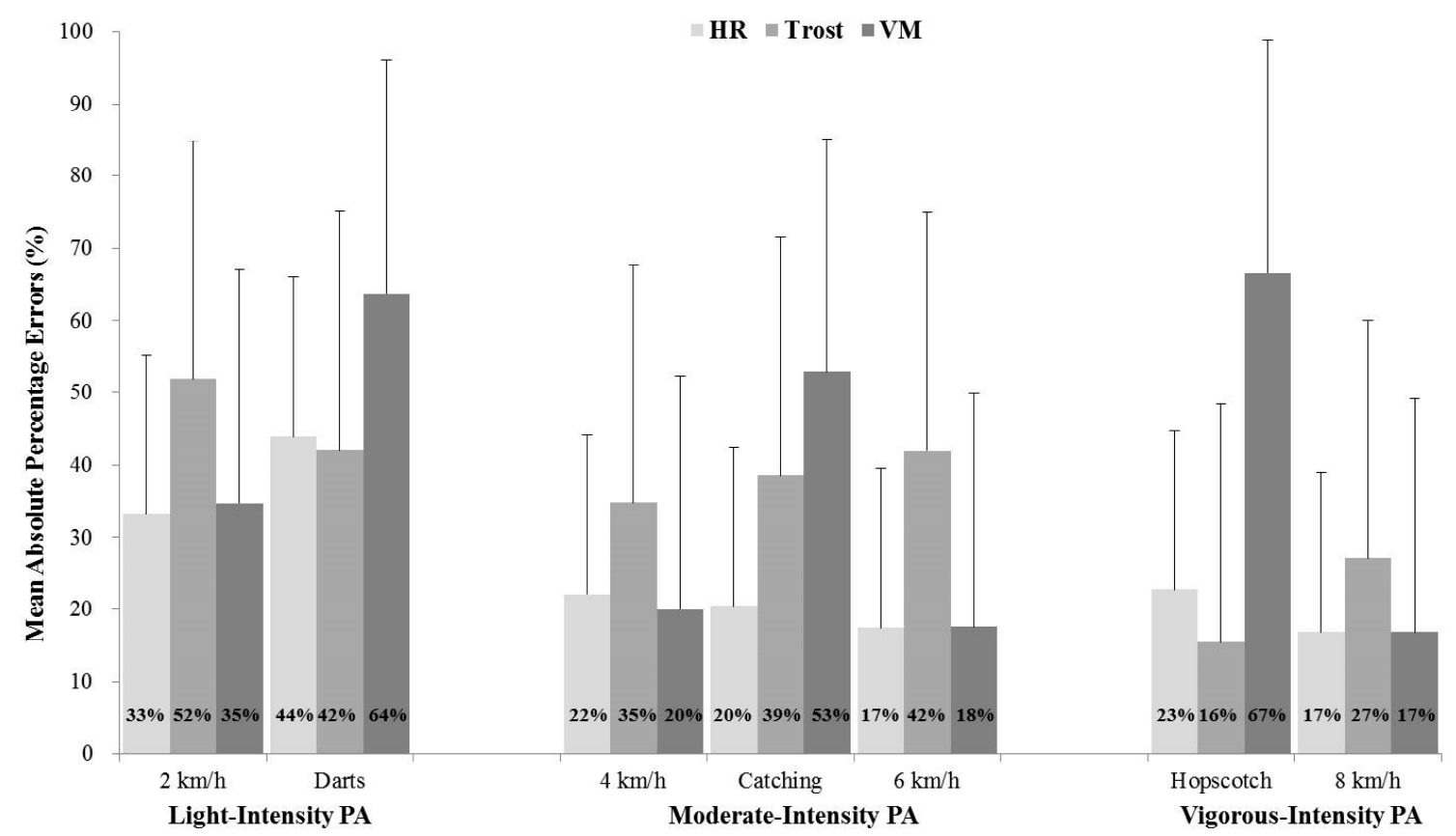

Figure 1. Mean Absolute Percentage Errors for energy expenditure (EE) estimated by heart rate (HR), Trost equation, and vector magnitudes (VM).

Figure 2 reveals the Bland-Altman plot for the extent of agreement between the criterion $\mathrm{EE}$ and the $\mathrm{EE}$ of each equation using the average difference values to examine the proportional systematic bias. The plots show $95 \%$ limits of agreement and lines of best fit. The heart-rate-based EE had the mean of the difference closer to zero (mean $=0.25$, difference $=4.75)$ compared to the accelerometer-based $\mathrm{EE}($ mean $=-1.38$, difference $=4.6)$ and Trost EE (mean $=1.04$, difference $=6.99$ ). The slopes for the fitted line indicated that values from the heart-rate-based equation and Trost equation have a tendency to increase in a negative direction, $($ slope $=-0.17$, intercept $=1.08)$, (slope $=-0.08$, intercept $=-1.09)$, respectively. However, the VM-based equation showed a tendency to increase in a positive direction (slope $=0.27$, intercept $=-0.34$ ).
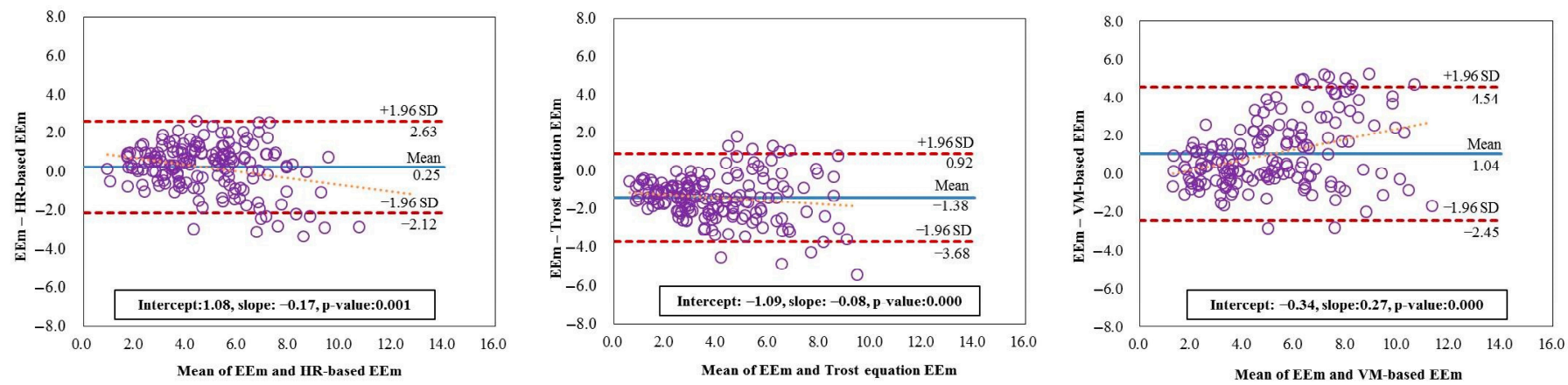

Figure 2. Bland-Altman plots for energy expenditure (EE) estimated by heart rate (HR), Trost equation, and vector magnitudes (VM).

The utility of equivalence testing made it possible to examine if the developed equations' estimates were equivalent to the estimate from the criterion EE measure (K4b2) (Figure 3). In the heart-rate-based EE, all activity values were completely included within the measured EE equivalence zone ( $\pm 10 \%$ of the criterion measurement). The VM-based EE values were overestimated beyond the equivalence zone in the dart activity (light-intensity activity), ball catching (moderate-intensity activity), and hopscotch (vigorous-intensity activities). The Trost equation-based EE values were underestimated in all activities except the hopscotch activity, outside of the equivalence zone. 

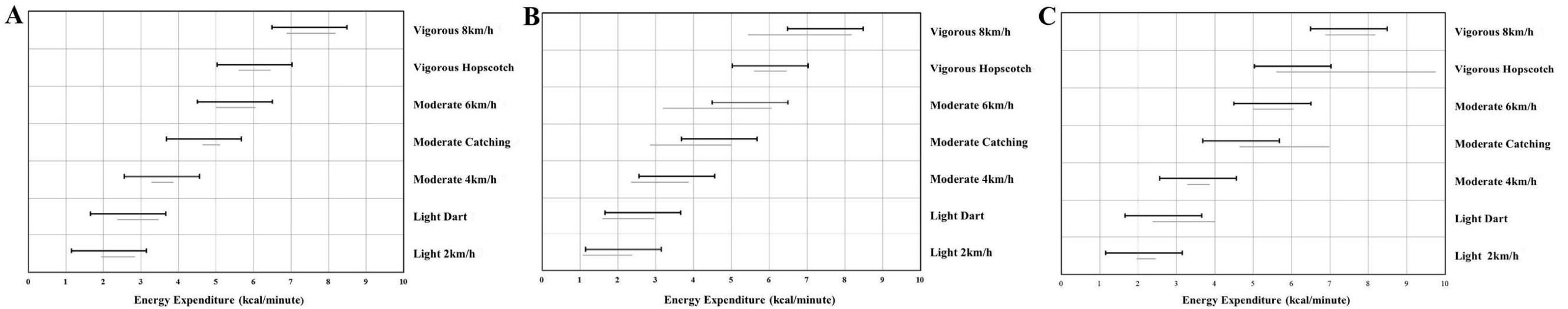

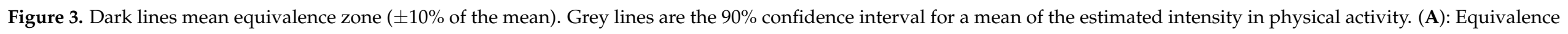

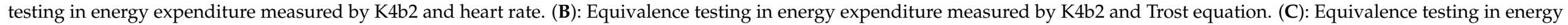
expenditure measured by $\mathrm{K} 4 \mathrm{~b} 2$ and vector magnitudes. 


\section{Discussion}

Objective measurement of EE in everyday life is a critical step in understanding the optimum mechanisms for preventing obesity and diseases in growing children. The present study was designed to examine the validity and utility of developing EE prediction equations using a heart rate monitor and commonly used accelerometers (i.e., ActiGraph). These devices are proven to be efficient tools for objectively monitoring PA intensity, frequency, and duration. However, there has been little research on PAEE equations for children. Furthermore, the ActiGraph accelerometer has been used by many researchers in order to present reference values for PA intensity cut-off points, but its use in the study of PAEE equations has been limited. The previously developed equation provides a valuable comparison since these devices have been considered the standard in most PAEE estimate studies. Therefore, the purpose of this study was to develop predictive PAEE equations by assessing heart rate responses and accelerometer counts while children are performing PA of light, moderate, and vigorous intensities to confirm the practical utility of the developed equations through cross validation.

The study results suggest that the accuracy of the prediction model seems improved when the HR-based prediction is used. PAEE estimates from this present study showed more than $80 \%$ of explanatory power in both the HR-based equation and VM-based equation. Moreover, the heart-rate-based EE estimates showed that PAEE estimates for all activities were included entirely within the equivalence zone. However, the VM-based PAEE results showed overestimation for throwing darts in light-intensity activity, ball catching in moderate-intensity activity, and hopscotch in vigorous-intensity activity. It has been reported that when an accelerometer is used as a physical activity assessment tool for children, it overestimates overground activities like throwing darts, ball catching, and hopscotch, but not treadmill activities [21]. These results are consistent with previous studies' findings that the PAEE estimate results using the accelerometer showed low accuracy in daily activities due to the effect from the upper and lower limb movements [33]. García-Prieto et al. [13] also investigated the accuracy of PAEE estimates in children's playground activities, and the study found that the HR-based estimates showed a higher correlation with the criterion method than accelerometers. The HRM has long been used as a tool to measure physical activity. In this regard, the development of a PAEE equation using heart rate will help improve the estimation accuracy of the PAEE in children.

Previous calibration studies [16,34-36] have developed equations for the accelerometer by using locomotor activities (i.e., walking and running), and the results generally showed a lower or moderate correlation compared to the measured EE. For instance, the Trost equation has been developed using treadmill walking and running, which significantly overestimated EE during normal and brisk walking. The weaker results for other activities are somewhat expected because it is impractical to expect an equation developed for locomotor activities to predict other activities with a similar validity. An advantage of the present study is that we included lifestyle activities and utilized the heart rate to develop the equation and improve the accuracy of the PAEE prediction equations, which may compromise the prediction of PAEE during walking and running. Some researchers suggest that the combination of HR and accelerometer overcomes PAEE estimates from the single device approach and can provide better predictive accuracy $[37,38]$; however, it requires more time to evaluate Flex-HR points during rest and the lowest HR during incremental exercise protocols such as a submaximal treadmill test. Based on the present study results, the HR-based EE prediction equation outperformed the Trost equation and VM-based equation. Therefore, our findings suggest that the HR-based prediction model's accuracy can be applied in field research settings.

Recently, personalized healthcare services using wearables are drawing attention in research and field applications. Additional functions such as blood pressure monitors and electrocardiograms in wearables have been installed and used as tools to help maintain and promote health. Wang et al. [39] investigated the accuracy of wrist-worn wearable devices on HR measurement and high correlations were observed in polar H7 ( $r=0.99)$, 
Apple Watch $(r=0.91)$, and Mio Fuse $(r=0.91)$. Dooley et al. [40] found that the Apple Watch and Fitbit Charge HR did not statistically show significant differences from the Polar heart rate monitor in light- and moderate-intensity exercise. The MAPE showed a valid HR measure for the Apple Watch from 1.14\% to 6.70\% and for the Fitbit Charge HR from $2.38 \%$ to $16.99 \%$. EE estimates from these devices over- or under-estimate depending on the activities included in the validity studies. Notably, high estimate errors were observed in light-intensity activities, and the EE estimates showed overestimation in free-living activities. To be specific, the Fitbit Flex model in wearables has been reported to be accurate in terms of information on sedentary behavior and total physical activity level, but not as accurate in classifying the light-intensity activities (92\% MAPE) and moderate to vigorous intensity activities (46\% MAPE) [41]. In addition, the accuracy of physical activity intensity classification provided from the Fitbit Charge HR, which now has the heart rate function added, is that the moderate- to vigorous-intensity activities showed a classification accuracy of $70.8 \%$. However, the accuracy for the light-intensity activities was $66.1 \%$, indicating more insufficient accuracy compared to the moderate- to vigorousintensity activities [42]. Although considerable research has been performed to develop and evaluate PAEE estimates, there still are no solid estimates for children's PAEE and many challenges remain in trying to develop standardized PAEE estimates. Therefore, it is believed that more accurate and diverse studies of children's PAEE measurements should be conducted based on this study.

Typically, those commercially available wearable trackers have targeted adults, however, interest in wearable trackers for children has been recently increasing. The wearable trackers have been applied in various research settings to promote physical activity in adolescents and children. To examine the utility of the trackers, several studies have examined the validity of the wearable trackers, but they found low validity for light PA and MVPA classification and reported a high error rate for EE estimates [41]. Since the accuracy of the built-in heart rate sensor in the wearable tracker has been significantly improved [43], the simple HR-based PAEE prediction equation developed in this study might be utilized in the wearable trackers for estimating PAEE to help researchers and healthcare providers examine children's PA.

This study has the following strengths and limitations. It developed an equation based on the heart rate and accelerometer, the representative objective testing tools among the PA assessment methods, and verified the field applicability through cross-validation. To our best knowledge, none of the studies confirmed that the HRM has higher predictive accuracy than accelerometers in various types of activities, and the EE prediction using heart rate can be used for the objective measurement of EE during moderate- to vigorous-intensity PA. However, the current study could not investigate the total PA level's accuracy because the cross-validation protocol was limited in each exercise intensity. Therefore, it is necessary to examine cross-validation reflecting an entire cycle of daily life in the same perspective. Furthermore, participants in the present study were limited to Korean adolescents and the number of boys in the study was higher than that of girls, so additional studies need to demonstrate more evidence with various ethnicities and balanced sample sizes.

The EE equation for children developed in this study emphasized practical applicability based on heart rate and an accelerometer. In particular, the energy expenditure equation using heart rate showed better accuracy than the equation using an accelerometer in terms of cross validity. These results may be utilized in physical activity intervention studies or pattern research in children. The present study's limitations include that regression analysis was performed based on the data obtained by repeatedly performing exercises of various intensities. Although this method has been used in many previous studies on the development of the exercise EE equations [15], there is a possibility of overestimating intervariable relations due to errors in data aggregation. Further studies are required to increase the number of samples in consideration of the hierarchical linear model and to develop an equation based on data obtained from participants of various characteristics (i.e., nationality, ages, and anthropometric information). 


\section{Conclusions}

This study describes the development and evaluation of heart-rate-based PAEE estimates in children. The study results indicated a higher accuracy PAEE estimate from the heart-rate-based measure than the accelerometer-based measure compared to objectively measuring EE. However, both the HRM and accelerometers revealed that light-intensity activities were likely to be overestimated. Therefore, the developed EE estimate equation should be carefully applied considering the research settings and population.

Author Contributions: Data curation, M.L., S.-K.K., J.-H.P., and J.-M.L.; formal analysis, J.-H.P. and J.-M.L.; investigation, M.L.; methodology, M.-W.S. and J.-M.L.; project administration, S.-K.K., and J.-M.L.; writing-original draft, M.L.; writing-review and editing, J.-H.P., M.-W.S. and J.-M.L. All authors have read and agreed to the published version of the manuscript.

Funding: This research received no external funding.

Institutional Review Board Statement: The study was conducted according to the guidelines of the Declaration of Helsinki, and approved by the Institutional Review Board of the University of Yong-in (IRB-2-1040966-AB-N-01-20-1404-HSR-010-1).

Informed Consent Statement: Informed consent was obtained from all participants and guardians involved in the study.

Data Availability Statement: The data presented in this study are available on request from the corresponding author.

Acknowledgments: The authors would like to thank all participants who participated in this study.

Conflicts of Interest: The authors no conflict of interest to declare.

\section{References}

1. Janssen, I.; Leblanc, A.G. Systematic review of the health benefits of physical activity and fitness in school-aged children and youth. Int. J. Behav. Nutr. Phys. Act. 2010, 7, 40. [CrossRef]

2. Anderson, E.; Durstine, J.L. Physical activity, exercise, and chronic diseases: A brief review. Sports Med. Health Sci. 2019, 1, 3-10. [CrossRef]

3. Granger, E.; Di Nardo, F.; Harrison, A.; Patterson, L.; Holmes, R.; Verma, A. A systematic review of the relationship of physical activity and health status in adolescents. Eur. J. Public Health 2017, 27 (Suppl. 2), 100-106. [CrossRef]

4. Byun, W.; Dowda, M.; Pate, R.R. Associations between screen-based sedentary behavior and cardiovascular disease risk factors in Korean youth. J. Korean Med. Sci. 2012, 27, 388-394. [CrossRef] [PubMed]

5. Piercy, K.L.; Troiano, R.P.; Ballard, R.M.; Carlson, S.A.; Fulton, J.E.; Galuska, D.A.; George, S.M.; Olson, R.D. The Physical Activity Guidelines for Americans. JAMA 2018, 320, 2020-2028. [CrossRef] [PubMed]

6. Herman, K.M.; Hopman, W.M.; Sabiston, C.M. Physical activity, screen time and self-rated health and mental health in Canadian adolescents. Prev. Med. 2015, 73, 112-116. [CrossRef] [PubMed]

7. Biddle, S.J.; Asare, M. Physical activity and mental health in children and adolescents: A review of reviews. Br. J. Sports Med. 2011, 45, 886-895. [CrossRef]

8. Sampasa-Kanyinga, H.; Hamilton, H.A.; Willmore, J.; Chaput, J.P. Perceptions and attitudes about body weight and adherence to the physical activity recommendation among adolescents: The moderating role of body mass index. Public Health 2017, 146, 75-83. [CrossRef] [PubMed]

9. Lang, C.; Brand, S.; Feldmeth, A.K.; Holsboer-Trachsler, E.; Pühse, U.; Gerber, M. Increased self-reported and objectively assessed physical activity predict sleep quality among adolescents. Physiol. Behav. 2013, 120, 46-53. [CrossRef]

10. Clark, S.L.; Denburg, M.R.; Furth, S.L. Physical activity and screen time in adolescents in the chronic kidney disease in children (CKiD) cohort. Pediatric Nephrol. 2016, 31, 801-808. [CrossRef] [PubMed]

11. Schmitz, K.H.; Treuth, M.; Hannan, P.; McMurray, R.; Ring, K.B.; Catellier, D.; Pate, R. Predicting energy expenditure from accelerometry counts in adolescent girls. Med. Sci. Sports Exerc. 2005, 37, 155-161. [CrossRef] [PubMed]

12. Stone, M.R.; Rowlands, A.V.; Eston, R.G. Relationships between accelerometer-assessed physical activity and health in children: Impact of the activity-intensity classification method. J. Sports Sci. Med. 2009, 8, 136-143. [PubMed]

13. García-Prieto, J.C.; Martinez-Vizcaino, V.; García-Hermoso, A.; Sánchez-López, M.; Arias-Palencia, N.; Fonseca, J.F.O.; MoraRodriguez, R. Energy Expenditure in Playground Games in Primary School Children Measured by Accelerometer and Heart Rate Monitors. Int. J. Sport Nutr. Exerc. Metab. 2017, 27, 467-474. [CrossRef]

14. Lyden, K.; Kozey, S.L.; Staudenmeyer, J.W.; Freedson, P.S. A comprehensive evaluation of commonly used accelerometer energy expenditure and MET prediction equations. Eur. J. Appl. Physiol. 2011, 111, 187-201. [CrossRef] 
15. Troiano, R.P.; McClain, J.J.; Brychta, R.J.; Chen, K.Y. Evolution of accelerometer methods for physical activity research. Br. J. Sports Med. 2014, 48, 1019-1023. [CrossRef] [PubMed]

16. Freedson, P.S.; Melanson, E.; Sirard, J. Calibration of the Computer Science and Applications, Inc. accelerometer. Med. Sci. Sports Exerc. 1998, 30, 777-781. [CrossRef] [PubMed]

17. Puyau, M.R.; Adolph, A.L.; Vohra, F.A.; Zakeri, I.; Butte, N.F. Prediction of activity energy expenditure using accelerometers in children. Med. Sci. Sports Exerc. 2004, 36, 1625-1631. [PubMed]

18. Treuth, M.S.; Schmitz, K.; Catellier, D.J.; McMurray, R.G.; Murray, D.M.; Almeida, M.J.; Going, S.; Norman, J.E.; Pate, R. Defining accelerometer thresholds for activity intensities in adolescent girls. Med. Sci. Sports Exerc. 2004, 36, 1259-1266.

19. Evenson, K.R.; Catellier, D.J.; Gill, K.; Ondrak, K.S.; McMurray, R.G. Calibration of two objective measures of physical activity for children. J. Sports Sci. 2008, 26, 1557-1565. [CrossRef] [PubMed]

20. Trost, S.G.; Ward, D.S.; Moorehead, S.M.; Watson, P.D.; Riner, W.; Burke, J.R. Validity of the computer science and applications (CSA) activity monitor in children. Med. Sci. Sports Exerc. 1998, 30, 629-633. [CrossRef] [PubMed]

21. Trost, S.G.; Way, R.; Okely, A.D. Predictive validity of three ActiGraph energy expenditure equations for children. Med. Sci. Sports Exerc. 2006, 38, 380-387. [CrossRef] [PubMed]

22. Ceesay, S.M.; Prentice, A.M.; Day, K.C.; Murgatroyd, P.R.; Goldberg, G.R.; Scott, W.; Spurr, G.B. The use of heart rate monitoring in the estimation of energy expenditure: A validation study using indirect whole-body calorimetry. Br. J. Nutr. 1989, 61, 175-186. [CrossRef] [PubMed]

23. De Groot, J.F.; de Jong, A.S.; Visser, T.; Takken, T. Validation of the Actical and Actiheart monitor in ambulatory children with spina bifida. J. Pediatr. Rehabil. Med. 2013, 6, 103-111. [CrossRef]

24. Calabró, M.A.; Stewart, J.M.; Welk, G.J. Validation of pattern-recognition monitors in children using doubly labeled water. Med. Sci. Sports Exerc. 2013, 45, 1313-1322. [CrossRef]

25. Harrell, J.S.; McMurray, R.G.; Baggett, C.D.; Pennell, M.L.; Pearce, P.F.; Bangdiwala, S.I. Energy costs of physical activities in children and adolescents. Med. Sci. Sports Exerc. 2005, 37, 329-336. [CrossRef]

26. Eston, R.G.; Rowlands, A.V.; Ingledew, D.K. Validity of heart rate, pedometry, and accelerometry for predicting the energy cost of children's activities. J. Appl. Physiol. 1998, 84, 362-371. [CrossRef]

27. McLaughlin, J.E.; King, G.A.; Howley, E.T.; Bassett, D.R., Jr.; Ainsworth, B.E. Validation of the COSMED K4 b2 portable metabolic system. Int. J. Sports Med. 2001, 22, 280-284. [CrossRef]

28. Gamelin, F.X.; Baquet, G.; Berthoin, S.; Bosquet, L. Validity of the polar S810 to measure R-R intervals in children. Int. J. Sports Med. 2008, 29, 134-138. [CrossRef]

29. Lee, J.M.; Byun, W. Comparison of Wearable Trackers' Ability to Estimate Sleep. Int. J. Environ. Res. Public Health 2018, 15, 1265. [CrossRef] [PubMed]

30. Park, J.H.; Seo, M.W. Let's Live Healthier: The Relationship between Suicidal Behavior and Physical Activity in an Age-, Gender-, and Body Mass Index-Matched Adults. Int. J. Environ. Res. Public Health 2020, 17, 8350. [CrossRef] [PubMed]

31. Kim, Y.; Crouter, S.E.; Lee, J.M.; Dixon, P.M.; Gaesser, G.A.; Welk, G.J. Comparisons of prediction equations for estimating energy expenditure in youth. J. Sci. Med. Sport 2016, 19, 35-40. [CrossRef]

32. Ferrar, K.; Evans, H.; Smith, A.; Parfitt, G.; Eston, R. A systematic review and meta-analysis of submaximal exercise-based equations to predict maximal oxygen uptake in young people. Pediatric Exerc. Sci. 2014, 26, 342-357. [CrossRef] [PubMed]

33. Trost, S.G.; Loprinzi, P.D.; Moore, R.; Pfeiffer, K.A. Comparison of accelerometer cut points for predicting activity intensity in youth. Med. Sci. Sports Exerc. 2011, 43, 1360-1368. [CrossRef] [PubMed]

34. Trost, S.G. Objective measurement of physical activity in youth: Current issues, future directions. Exerc. Sport Sci. Rev. 2001, 29, 32-36. [CrossRef]

35. Trost, S.G.; Kerr, L.M.; Ward, D.S.; Pate, R.R. Physical activity and determinants of physical activity in obese and non-obese children. Int. J. Obes. 2001, 25, 822-829. [CrossRef] [PubMed]

36. Trost, S.G.; Pate, R.R.; Sallis, J.F.; Freedson, P.S.; Taylor, W.C.; Dowda, M.; Sirard, J. Age and gender differences in objectively measured physical activity in youth. Med. Sci. Sports Exerc. 2002, 34, 350-355. [CrossRef]

37. Yang, L.; Lu, K. Evaluation of physiological workload assessment methods using heart rate and accelerometry for a smart wearable system. Ergonomics 2019, 62, 694-705. [CrossRef]

38. Hashiguchi, N.; Kodama, K.; Lim, Y. Practical Judgment of Workload Based on Physical Activity, Work Conditions, and Worker's Age in Construction Site. Sensors 2020, 20, 3786. [CrossRef]

39. Wang, R.; Blackburn, G.; Desai, M.; Phelan, D.; Gillinov, L.; Houghtaling, P.; Gillinov, M. Accuracy of Wrist-Worn Heart Rate Monitors. JAMA Cardiol. 2017, 2, 104-106. [CrossRef] [PubMed]

40. Dooley, E.E.; Golaszewski, N.M. Estimating Accuracy at Exercise Intensities: A Comparative Study of Self-Monitoring Heart Rate and Physical Activity Wearable Devices. JMIR mHealth uHealth 2017, 5, e34. [CrossRef]

41. Byun, W.; Lee, J.M.; Kim, Y.; Brusseau, T.A. Classification Accuracy of a Wearable Activity Tracker for Assessing Sedentary Behavior and Physical Activity in 3-5-Year-Old Children. Int. J. Environ. Res. Public Health 2018, 15, 594. [CrossRef] [PubMed]

42. Kang, S.; Kim, Y.; Byun, W.; Suk, J.; Lee, J.M. Comparison of a Wearable Tracker with Actigraph for Classifying Physical Activity Intensity and Heart Rate in Children. Int. J. Environ. Res. Public Health 2019, 16, 2663. [CrossRef] [PubMed]

43. Støve, M.P.; Holm, R.S.; Kjaersgaard, A.S.; Duncker, K.; Jensen, M.R.; Larsen, B.T. Measurement latency significantly contributes to reduced heart rate measurement accuracy in wearable devices. J. Med. Eng. Technol. 2020, 44, 125-132. [CrossRef] [PubMed] 